3. Accelerated Partner Therapy (APT Pharmacy): nurse initiated $\mathrm{PN}$ at the general practise followed by assessment of sex partner by trained community pharmacist;

4. Patient referral, where patients are advised by phone by qualified health adviser on the need for partner to be tested and treated.

5. Provider referral, where patients accept the offer of a specialist health adviser contacting one or more partner(s) by phone.

For all pathways primary costs were collected prospectively in a specific exploratory study.

Results The least costly strategy is nurse led PN (strategy 2) costing approximately $£ 53$ per index case (2011 costs). The most costly strategy is provider referral (strategy 6) which cost $£ 96$ per index case.

Conclusion Where health service providers assume responsibility for contacting partners there will be substantial additional cost. Before any such policy is implemented, a demonstrable improvement in PN outcomes should be established.

\section{P6.061 DEVELOPMENT OF A VALIDATED QUESTIONNAIRE FOR HIV ATTENDEES}

doi:10.1136/sextrans-2013-051184.1214

'L Land, ${ }^{2}$ S Sizmur, ${ }^{3} \mathrm{~J}$ Harding, ${ }^{3} \mathrm{~J}$ D C Ross. 'Birmingham City University, Birmingham, UK; ${ }^{2}$ Picker Institute, Europe, UK; ${ }^{3}$ Whittall Street Clinic, Birmingham, UK

Background Patients are becoming more actively involved in decisions about their care and have greater influence to change and improve the quality of services by reporting their experiences. Within HIV services, positive experiences increase engagement with services and have been linked to higher levels of treatment adherence. A previous systematic review assessing satisfaction with care failed to locate a gold standard method of measuring satisfaction in this setting.

Aim to design a specific HIV patient satisfaction questionnaire

Methods Four work streams were employed to develop and test a new questionnaire. Firstly, key themes identified in the systematic review were used as a topic guide for focus group discussion to assess their relevance and importance. Four focus groups comprising 32 participants were conducted and revealed the importance of physician knowledge and expertise; dignity, autonomy and respect; and good communication. The second stream involved interviews with ten patients, exploring their motivation to complete a questionnaire. Thirdly, data from the focus groups and interviews were used to develop an initial questionnaire which was cognitively tested on a further ten patients, this provided face validity for the questionnaire design, layout and wording. The final stream employed a pilot study of the questionnaire with 80 clinic attendees.

Results The pilot survey demonstrated that there was a high completion rate. Two questions were modified and additional routing instructions were added. Pairwise correlations reflected the thematic structure of the questionnaire and supported good criterion validity.

Conclusions The combination of a systematic analysis of previous patient survey tools, focus group discussions and cognitive testing of the questionnaire was used to ensure high content validity. The questionnaire was found to be acceptable to patients and a high completion rate was attained without the use of a financial incentive.

\section{P6.062 OUALIAIDS: QUALITY OF AMBULATORY HEALTH SERVICES WHO ATTEND PEOPLE LIVING WITH HIV/AIDS IN BRAZIL}

doi:10.1136/sextrans-2013-051184.1215

F M Lazarini, J Uesono, M A Freitas, D B Greco. Ministry of Health, Brazil, Department of STD, Aids and Viral Hepatitis, Brasília, Brazil
Background Between 2007 and 2012, there was an increase of 14\% of Specialized Care Services for people living with HIV/AIDS (PLHIV), totaling 724. A decade ago the Qualiaids evaluates the quality of outpatient care in public services in Brazil. The aim of this study is to describe the dimensions of this evaluation method, the highlights of care services and its importance in monitoring the quality of care for PLHIV.

Methods Were made three national applications: one manual and two online through a questionnaire completed by the services. Each question has a score, and there were grouped under three components: availability of services inputs, organisation of care delivery and managerial aspects. To rank the services Were used the average obtained in the questions and then were grouped by technique of $\mathrm{K}$-mean. The analysis resulted in the layering of five groups in decreasing levels of quality. The online tool also released a set of recommendations for good practises in order to elucidate the quality criteria that support the score of each question.

Results Qualiaids covered $80 \%$ of the services in 2007 and $90 \%$ in 2010. Increased the overall average and the three dimensions of quality, with statistical significance for most of them. The size of management remains the dimension with the lowest quality rating. The great majority of responses to the questionnaire QUALIAIDS was maintained in similar frequencies (variations less than 10\%) among the respondents of the two sets of ratings.

Conclusion From the self assessment were planned the feedback workshops to improving the quality to knowing the difficulties and systematise the priorities for the development of an action plan customised for each service. This process favoured the implementation of agreements and pacts that allowed monitoring of quality indicators and subsidised practises to improve the quality of care services.

\section{P6.063 VERTICAL TRANSMISSION OF HIV BY AGE OF INFANT TESTING AND TYPE OF MOTHER/INFANT PROPHYLAXIS IN TIGRAY, ETHIOPIA}

doi:10.1136/sextrans-2013-051184.1216

${ }^{1}$ T A Kahsu, ${ }^{2} \mathrm{M}$ Abraha, ${ }^{2} \mathrm{~F}$ Tadesse, ${ }^{2} \mathrm{~B}$ Crandall, ${ }^{3} \mathrm{H}$ Godefay, ${ }^{1} \mathrm{E} \mathrm{L} \mathrm{L}$ Konings. 'Management Sciences for Health, Addis Ababa, Ethiopia, ${ }^{2} \mathrm{MSH}$, Addis Ababa, Ethiopia, ${ }^{3}$ Tigray Regional Health Bureau, Mekele, Ethiopia

Background Following a number of other African countries, Ethiopia has recently adopted option $\mathrm{B}+$ as the national PMTCT standard. Option B + puts all HIV-positive pregnant women on HAART for life, regardless of the CD4 count. This study examines whether HAART for maternal prophylaxis resulted in lower vertical HIV transmission in Tigray.

Methods We used data from Tigray's regional DBS data base to calculate HIV infection rates by PMTCT regimen in all HIV-exposed infants (HEIs) tested by December 2012. We calculated the relative risk (RR) of having a HIV + baby by age of testing and the mother's PMTCT regimen.

Results There was a significant difference in HIV rates between babies tested at $0-2$ months ( $4 \%)$ and $2-8$ months $(9 \%)(R R=0.43$ $\mathrm{CI}=0.28 .0 .65 ; \mathrm{p}=0.0001)$. HIV infection was significantly higher in babies tested past 8 months $(16 \%$ ) (respectively, $R R=0.23$, $\mathrm{CI}=0.14 .0 .38 ; \mathrm{p}<0.0001$ and $\mathrm{RR}=0.55, \mathrm{CI}=0.36 .0 .86 ; \mathrm{p}=0.005$ ) Maternal prophylaxis was a significant factor with a further significant difference between HAART $(2 \%)$ and dual prophylaxis $(14 \%)$ $(\mathrm{RR}=0.37 ; \mathrm{CI}=0.19 .0 .74 ; \mathrm{p}=0.006)$.

Conclusions This study underscores the importance of putting HIV-positive pregnant mothers on PMTCT, especially HAART, and promoting institutional delivery and early infant testing. HAART was the most effective PMTCT regimen, regardless of the infant's regimen. This finding is particularly important in Ethiopia where most women give birth at home and may not be able to give the right medication to their baby at the right time. This data strongly 